\title{
Consideration of longitudinal vibration of automobiles in planar model with taking road deformation and loss of contact into account
}

\author{
Ham Vu Cong ${ }^{1}$, Cuong Phung Manh ${ }^{2}$, Dung Tran Quang ${ }^{3}$ \\ Faculty of Mechanics, Le Quy Don Technical University, Ha Noi, Vietnam \\ ${ }^{2}$ Corresponding author \\ E-mail: ${ }^{1}$ conghamf767@gmail.com, ${ }^{2}$ phungmanhcuongk23@gmail.com, ${ }^{3}$ tranquangdung79@gmail.com \\ Received 3 July 2020; received in revised form 11 December 2020; accepted 14 January 2021 \\ DOI https://doi.org/10.21595/jve.2021.21575 \\ Check for updates \\ Copyright (C) 2021 Ham Vu Cong, et al. This is an open access article distributed under the Creative Commons Attribution License, which \\ permits unrestricted use, distribution, and reproduction in any medium, provided the original work is properly cited.
}

\begin{abstract}
This article considers longitudinal planar vibration of a two-axle automobile moving linearly with a constant velocity and subjected to pre-deterministic kinematic excitation caused by the rough road surface. The automobile is modeled as a vibration system which has three masses and four degrees of freedom. The deformed road is modeled as an elastic beam which is simply supported at the two ends and lying on Kelvin's visco-elastic ground. The change in dimensions of the contact areas is considered. The loss of contact between the wheels and the road surface is taken into account by producing the contact state parameters in the differential equations of motion of the vibration system. The partial differential equation which describes the motion of deformed road is then transformed into a set of all ordinary differential equations by applying the Bubnov-Galerkin's method. Some typical results coming from numerical consideration are also presented in the article.
\end{abstract}

Keywords: vibration, automobile, planar model, half-car model, wheel separation, loss of contact, road deformation.

\section{Introduction}

When an automobile moves on rough roads, vertical vibrations appear as an inherent property. If the level of vibration exceeds a definite threshold, the wheels of the automobile may separate from the road and this phenomenon is called as the loss of contact, or the wheel separation in some documents. The loss of contact reduces controllability of the automobile both in velocity and direction, and therefore, the safety of movement.

In many books and papers concerned with the vibration of automobiles, the loss of contact is either disregarded such as in the references from [1-13] or although regarded but all spring-damper couples which represent the wheels has the lower end consistently clamped to the road surface as in [14]. These physical models are widely applied and help scientists to gain a lot of significant results, but obviously not authentic. It is needed to propose other models which have the authenticity higher.

Recently, the authors of this own article have started investigating into vertical vibration of automobiles with taking account of wheel separation or/and road deformation. The loss of contact is taken into account by introducing the so-called contact state parameters while the road deformation is taken into account by applying the models of elastic beam or rectangular plate on Kelvin's visco-elastic ground. In papers $[15,16]$, the one-fourth model is applied and the loss of contact is taken into account, but only the article [16] takes account of the road deformation. In the article [17], longitudinal vibration of automobiles is considered in the planar model (a half-car or one-second model), the loss of contact is also taken into account, but the road deformation is not regarded. This paper continues to consider longitudinal vibration of automobiles in the planar model where the loss of contact is taken into account by using two contact state parameters and the road deformation is taken into account by applying the models of the elastic beam on Kelvin's visco-elastic ground. 


\section{Formulation of the problem}

\subsection{Assumptions}

Vibration model of the vehicle-road coupled system is made with these assumptions:

- Automobile under consideration has the body and two axles absolutely rigid and move linearly with a constant velocity.

- Dynamic behavior of all spring-damper couples in the systems is linear.

- Contact area (if exists) between each wheel and the road surface is a rectangle whose dimension in the direction of vehicle axle is unchanged while loaded.

- The road profile is assumed to be predeterministic.

\subsection{Contact characteristics between the wheel and the road}

Fig. 1 shows a round wheel that is lying in contact state with the road surface and its vibration model. In the figure, $r_{w}$ is the radius of the wheel; $b_{L}$ - the width of the tyre, also the width of the contact area if exists; $k_{L}, c_{L}$ - the wheel string-damper couple which represents the dynamic behavior of the wheel in vertical direction; $C$ - center of the wheel; $Q$ - vertical load applied on the wheel axle; $R$ - reaction force from the road; $\Delta z_{L}$ - vertical compress deformation of the wheel or the spring representing its elasticity; $d_{c}$ - the length of the contact area; $D$ - the expected contact point between the road surface and the wheel. In general, $D$ is the shadow (in vertical direction) of the wheel center onto the road surface; if the contact state between the wheel and the road surface exists, $D$ is also the center of the contact area.

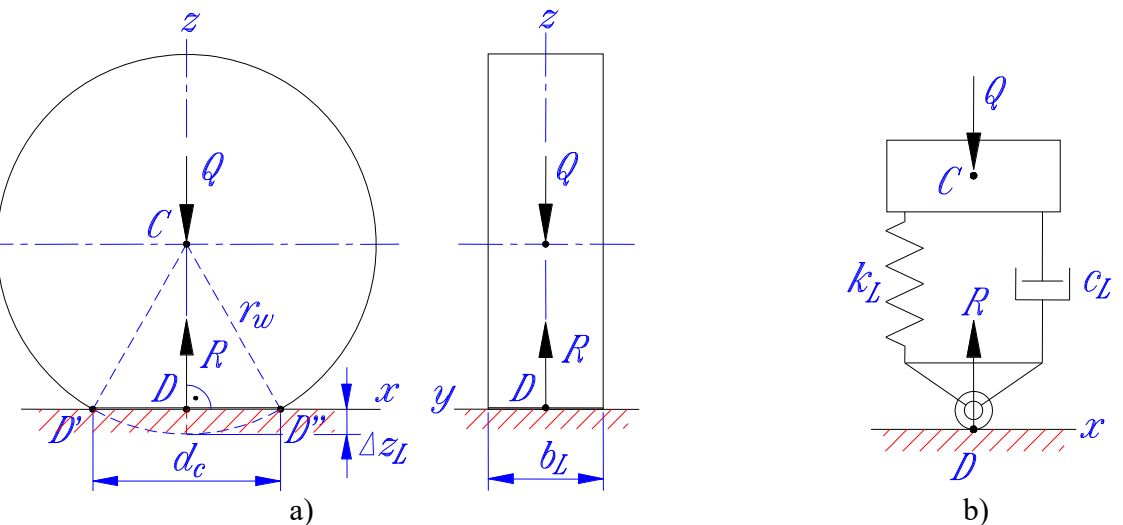

Fig. 1. a) Contact characteristics and b) vibration model of a deformed wheel

Assuming that the off-contact-area part of the wheel profile is still exactly rounded with the radius unchanged, we can get the relation between the length of contact area and vertical deformation of the wheel:

$d_{c}=2 \sqrt{r_{w}^{2}-\left(r_{w}-\Delta z_{L}\right)^{2}}$.

Let $F_{L}$ be the resultant of spring and damping forces in the wheel spring-damper couple. From the equilibrium condition of forces acting in the wheel model one can get $Q=R=F_{L}$. The value of $F_{L}$ can be expressed in term of vertical compress deformation of the wheel $\Delta z_{L}\left(\Delta z_{L}\right.$ is also the spring which represents the wheel) as:

$F_{L}=k_{L}\left(\Delta z_{L}\right)+c_{L} \frac{d\left(\Delta z_{L}\right)}{d t}$. 
If the loss of contact really appears or starts to appear, the wheel is not deformed and the values of $\Delta z_{L}, d_{c}$ and $F_{L}$ are all equal to zero.

Now if we redefine $\Delta z_{L}$ as the difference in vertical displacements of points $D$ and $C$, i.e. $\Delta z_{L}=u_{D}-u_{C}$, noting that both road deformation and wheel separation are taken into account, then the expression in the right side of Eq. (2) can be used to verify if the loss of contact occurs or not. This expression can be called as the verifying value of contact force and denoted as $\bar{F}_{L}$, so that:

$\bar{F}_{L}=k_{L}\left(u_{D}-u_{C}\right)+c_{L}\left(\dot{u}_{D}-\dot{u}_{C}\right)$.

It is obvious that if $\bar{F}_{L} \geq 0$, the wheel still lies in the contact state with the road and $\bar{F}_{L}$ is also the resultant force of the wheel spring-damper couple. If $\bar{F}_{L}<0$, the wheel separates from the road and the resultant force of the wheel spring-damper couple is equal to zero. According to this reasoning, we introduce the so-called contact state parameter which is denoted as $s$ and taken value as $s=1$ if $\bar{F}_{L} \geq 0$ and $s=0$ if $\bar{F}_{L}<0$. The formula for the resultant force of the wheel spring-damper couple in all three different states of relative position of the wheel-road couple (really contacting, starting to separate or contact, and really losing contact) now can be uniquely written as follows:

$F_{L}=s \bar{F}_{L}=s\left[k_{L}\left(u_{D}-u_{C}\right)+c_{L}\left(\dot{u}_{D}-\dot{u}_{C}\right)\right]$.

In order to distinguish the front and the rear wheels later, we will use subscripts or superscripts "1" and " 2 " respectively, such as $k_{L j}, c_{L j}, F_{L j}, s_{j}, d_{c j}, \Delta z_{L j}$, etc. $(j=1,2)$.

\subsection{Vibration model of the vehicle-road coupled system}

Basing on the construction of two-axle automobiles and the assumptions mentioned above, we can make the longitudinal planar vibration model of an automobile taking road deformation into account as shown in Fig. 2. The vehicle is modeled as a vibration system of three masses corresponding to the body and the two axles while the deformable road is modeled as an homo-geneous elastic beam lying on the Kelvin's visco-elastic ground. Fig. 2 describes the mechanical system under consideration in the so-called natural position in which all the springs are completely free and the road lies in the contact state with the two wheels.

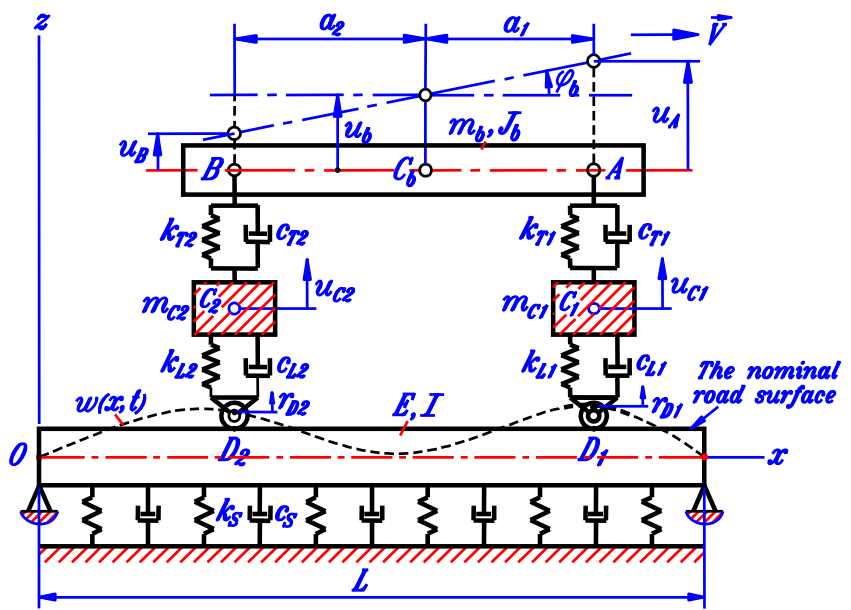

Fig. 2. Longitudinal vibration planar model of the vehicle-road coupled system

Here are some explanations for Fig. 2: 
$C_{b}, C_{1}, C_{2}$ - centers of gravity of the body, the front and the rear axles, respectively;

$A, B$ - connection points of suspension spring-damper couples to the vehicle body;

$m_{b}, J_{b}, m_{C 1}, m_{C 2}$ - inertial characteristics of the vehicle body and the two axles;

$\left\{k_{T 1}, c_{T 1}\right\},\left\{k_{T 2}, c_{T 2}\right\}$ - suspension spring-damper couples at the front and the rear axles;

$\left\{k_{L 1}, c_{L 1}\right\},\left\{k_{L 2}, c_{L 2}\right\}$ - wheel spring-damper couples at the front and the rear axles;

$\left\{k_{S}, c_{S}\right\}$ - stiffness and damping coefficient of Kelvin's visco-elastic ground;

$a_{1}, a_{2}$ - distances in horizontal direction from the center of gravity of the vehicle body to the front and the rear axles;

$w=w(x, t)-$ displacement function of the beam representing the deformed road;

$u_{b}, \varphi_{b}$ - vertical and angular displacements of the vehicle body;

$u_{C 1}, u_{C 2}$ - vertical displacements of the front and the rear axles;

$u_{A}, u_{B}$ - vertical displacements of points $A$ and $B$;

$r_{D 1}, r_{D 2}$ - representatives of the heights (or depths) at the expected contact points $D_{1}, D_{2}$ from the nominal road surface due to the road roughness.

It is noted that the displacements $u_{b}, \varphi_{b}, u_{C 1}, u_{C 2}$ are measured from the natural position of three masses (vehicle body and two axles).

\subsection{Determination of forces acting on the automobile}

Fig. 3 shows the force diagrams of three masses in vibration model after freeing them. In the figure, $G_{b}, G_{C 1}, G_{C 2}$ are the gravitational forces of three masses; $F_{T 1}, F_{T 2}, F_{T 1}^{\prime}, F_{T 2}^{\prime}$ - the resultant forces of the suspension spring-damper couples; $F_{L 1}, F_{L 2}$ - the resultant forces of the wheel springdamper couples, or the contact forces for simpliciy. Subscripts $b, 1$ and 2 used here imply that the concerned quantity belongs to or concerns with the vehicle body, the front and the rear axles, respectively.

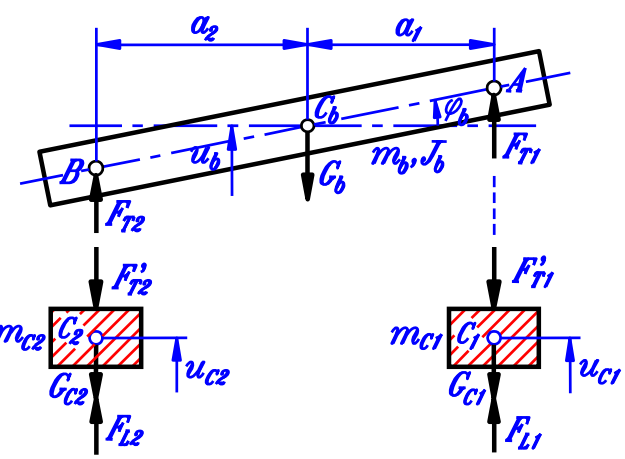

Fig. 3. Force diagrams of vehicle body and two axles

The forces in Fig. 3 can be expressed as:

$G_{b}=m_{b} g, \quad G_{C 1}=m_{C 1} g, \quad G_{C 2}=m_{C 2} g$,

$F_{T 1}=F_{T 1}^{\prime}=k_{T 1}\left(u_{C 1}-u_{A}\right)+c_{T 1}\left(\dot{u}_{C 1}-\dot{u}_{A}\right)$,

$F_{T 2}=F_{T 2}^{\prime}=k_{T 2}\left(u_{C 2}-u_{B}\right)+c_{T 2}\left(\dot{u}_{C 2}-\dot{u}_{B}\right)$,

$F_{L 1}=s_{1} \bar{F}_{L 1}=s_{1}\left[k_{L 1}\left(u_{D 1}-u_{C 1}\right)+c_{L 1}\left(\dot{u}_{D 1}-\dot{u}_{C 1}\right)\right]$,

$F_{L 2}=s_{2} \bar{F}_{L 2}=s_{2}\left[k_{L 2}\left(u_{D 2}-u_{C 2}\right)+c_{L 2}\left(\dot{u}_{D 2}-\dot{u}_{C 2}\right)\right]$.

Vertical displacements $u_{A}, u_{B}, u_{D 1}, u_{D 2}$ in Eq. (5) can be calculated as:

$u_{A}=u_{b}+a_{1} \varphi, \quad u_{B}=u_{b}-a_{2} \varphi, \quad u_{D 1}=w_{D 1}+r_{D 1}, \quad u_{D 2}=w_{D 2}+r_{D 2}$,

where $w_{D 1}=w(x, t)\left\|_{x=x_{D 1}}, w_{D 2}=w(x, t)\right\|_{x=x_{D 2}}$ are vertical displacements at points $D_{1}$ and 
$D_{2}$ of the beam which represents the deformed road.

Substituting Eq. (6) into Eq. (5) one can get the expressions of resultant forces in suspension and wheel spring-damper couples:

$$
\begin{aligned}
& F_{T 1}=F_{T 1}^{\prime}=k_{T 1}\left[u_{C 1}-\left(u_{b}+a_{1} \varphi_{b}\right)\right]+c_{T 1}\left[\dot{u}_{C 1}-\left(\dot{u}_{b}+a_{1} \dot{\varphi}_{b}\right)\right], \\
& F_{T 2}=F_{T 2}^{\prime}=k_{T 2}\left[u_{C 2}-\left(u_{b}-a_{2} \varphi_{b}\right)\right]+c_{T 2}\left[\dot{u}_{C 2}-\left(\dot{u}_{b}-a_{2} \dot{\varphi}_{b}\right)\right], \\
& F_{L 1}=s_{1}\left[k_{L 1}\left(w_{D 1}+r_{D 1}-u_{C 1}\right)+c_{L 1}\left(\dot{w}_{D 1}+\dot{r}_{D 1}-\dot{u}_{C 1}\right)\right], \\
& F_{L 2}=s_{2}\left[k_{L 2}\left(w_{D 2}+r_{D 2}-u_{C 2}\right)+c_{L 2}\left(\dot{w}_{D 2}+\dot{r}_{D 2}-\dot{u}_{C 2}\right)\right] .
\end{aligned}
$$

\subsection{Differential equations of motion of the mechanical system}

\subsubsection{Differential equations of motion of the vehicle}

By writing the dynamic equations of three masses in Fig. 3 then using the Eq. (7) of forces and taking some needed arrangements, we can get the differential equations of motion of the vehicle as follows:

$$
\left\{\begin{array}{l}
m_{b} \ddot{u}_{b}+\left(c_{T 1}+c_{T 2}\right) \dot{u}_{b}+\left(c_{T 1} a_{1}-c_{T 2} a_{2}\right) \dot{\varphi}_{\mathrm{b}}-c_{T 1} \dot{u}_{C 1}-c_{T 2} \dot{u}_{C 2} \\
\quad+\left(k_{T 1}+k_{T 2}\right) u_{b}+\left(k_{T 1} a_{1}-k_{T 2} a_{2}\right) \varphi_{b}-k_{T 1} u_{C 1}-k_{T 2} u_{C 2}=-m_{b} g, \\
J_{b} \ddot{\varphi}_{b}+\left(c_{T 1} a_{1}-c_{T 2} a_{2}\right) \dot{u}_{b}+\left(c_{T 1} a_{1}^{2}+c_{T 2} a_{2}^{2}\right) \dot{\varphi}_{b}-c_{T 1} a_{1} \dot{u}_{C 1}+c_{T 2} a_{2} \dot{u}_{C 2} \\
\quad+\left(k_{T 1} a_{1}-k_{T 2} a_{2}\right) u_{b}+\left(k_{T 1} a_{1}^{2}+k_{T 2} a_{2}^{2}\right) \varphi_{b}-k_{T 1} a_{1} u_{C 1}+k_{T 2} a_{2} u_{C 2}=0, \\
m_{C 1} \ddot{u}_{C 1}-c_{T 1} \dot{u}_{b}-c_{T 1} a_{1} \dot{\varphi}_{b}+\left(c_{T 1}+s_{1} c_{L 1}\right) \dot{u}_{C 1}-k_{T 1} u_{b}-k_{T 1} a_{1} \varphi_{b} \\
\quad+\left(k_{T 1}+s_{1} k_{L 1}\right) u_{C 1}=-m_{C 1} g+s_{1}\left[k_{L 1}\left(w_{D 1}+r_{D 1}\right)+c_{L 1}\left(\dot{w}_{D 1}+\dot{r}_{D 1}\right)\right], \\
m_{C 2} \ddot{u}_{C 2}-c_{T 2} \dot{u}_{b}+c_{T 2} a_{2} \dot{\varphi}_{b}+\left(c_{T 2}+s_{2} c_{L 2}\right) \dot{u}_{C 2}-k_{T 2} u_{b}+k_{T 2} a_{2} \varphi_{b} \\
\quad+\left(k_{T 2}+s_{2} k_{L 2}\right) u_{C 2}=-m_{C 2} g+s_{2}\left[k_{L 2}\left(w_{D 2}+r_{D 2}\right)+c_{L 2}\left(\dot{w}_{D 2}+\dot{r}_{D 2}\right)\right] .
\end{array}\right.
$$

\subsubsection{Differential equation of motion of the deformed road}

As presented above, the deformed road is modeled as homogeneous elastic beam that lies on Kelvin's visco-elastic ground. Additionally, the beam is simply supported at the two ends has the length of $L$, the rectangular cross-section with the width $b_{B}$ and the height $h_{B}$. Vertical displacement of the beam is a function of the $x$-coordinate and time $t$, ie. $w=w(x, t)$.

By considering the equilibrium of a typical beam element, we can obtain the differential equation of motion of the beam which represents the deformed road as follows [16]:

$\rho h_{B} \frac{\partial^{2} w(x, t)}{\partial t^{2}}+c_{S} \frac{\partial w(x, t)}{\partial t}+k_{S} w(x, t)+\frac{E I}{b_{B}} \frac{\partial^{4} w(x, t)}{\partial x^{4}}+p(x, t)=-\rho h_{B} g$.

In Eq. (9), $\rho$ and $E$ - mass density and Young's module of beam material; $I$ - bending inertial moment of beam cross-section $\left(I=b_{B} h_{B}^{3} / 12\right) ; g$ - gravitational acceleration and $p(x, t)-$ function of pressure distribution in the contact areas. Function $p(x, t)$ is assumed unchanged in $y$-direction and exists in wheel-beam contact areas only.

Solution $w=w(x, t)$ of Eq. (9) should be satisfied the boundary conditions:

$w(x, t)\left\|_{x=0}=w(x, t)\right\|_{x=L}=0$.

The differential equations of motion of the vehicle-road coupled system are the combination of four ordinary differential Eq. (8) and partial differential Eq. (9). 


\subsection{Simpler particular cases of the differential equations of motion}

\subsubsection{The case of ignoring road deformation}

In case the road deformation is ignored, we have $w(x, t)=0$ for all $x$ and $t$. Moreover, it can be assumed that $k_{S}=c_{S}=\infty$ and Eq. (9) becomes an identity. The differential equations of motion of the vehicle-road coupled system reduce to those of the vehicle only and have the form as follows:

$$
\left\{\begin{array}{l}
m_{b} \ddot{u}_{b}+\left(c_{T 1}+c_{T 2}\right) \dot{u}_{b}+\left(c_{T 1} a_{1}-c_{T 2} a_{2}\right) \dot{\varphi}_{b}-c_{T 1} \dot{u}_{C 1}-c_{T 2} \dot{u}_{C 2} \\
\quad+\left(k_{T 1}+k_{T 2}\right) u_{b}+\left(k_{T 1} a_{1}-k_{T 2} a_{2}\right) \varphi_{b}-k_{T 1} u_{C 1}-k_{T 2} u_{C 2}=-m_{b} g \\
J_{b} \ddot{\varphi}_{b}+\left(c_{T 1} a_{1}-c_{T 2} a_{2}\right) \dot{u}_{b}+\left(c_{T 1} a_{1}^{2}+c_{T 2} a_{2}^{2}\right) \dot{\varphi}_{b}-c_{T 1} a_{1} \dot{u}_{C 1}+c_{T 2} a_{2} \dot{u}_{C 2} \\
\quad+\left(k_{T 1} a_{1}-k_{T 2} a_{2}\right) u_{b}+\left(k_{T 1} a_{1}^{2}+k_{T 2} a_{2}^{2}\right) \varphi_{b}-k_{T 1} a_{1} u_{C 1}+k_{T 2} a_{2} u_{C 2}=0 \\
m_{C 1} \ddot{u}_{C 1}-c_{T 1} \dot{u}_{b}-c_{T 1} a_{1} \dot{\varphi}_{b}+\left(c_{T 1}+s_{1} c_{L 1}\right) \dot{u}_{C 1}-k_{T 1} u_{b}-k_{T 1} a_{1} \varphi_{b} \\
\quad+\left(k_{T 1}+s_{1} k_{L 1}\right) u_{C 1}=-m_{C 1} g+s_{1}\left(k_{L 1} r_{D 1}+c_{L 1} \dot{r}_{D 1}\right) \\
\quad m_{C 2} \ddot{u}_{C 2}-c_{T 2} \dot{u}_{b}+c_{T 2} a_{2} \dot{\varphi}_{b}+\left(c_{T 2}+s_{2} c_{L 2}\right) \dot{u}_{C 2}-k_{T 2} u_{b}+k_{T 2} a_{2} \varphi_{b} \\
\quad+\left(k_{T 2}+s_{2} k_{L 2}\right) u_{C 2}=-m_{C 2} g+s_{2}\left(k_{L 2} r_{D 2}+c_{L 2} \dot{r}_{D 2}\right) .
\end{array}\right.
$$

\subsubsection{The case of disregarding the loss of contact}

If the wheels are assumed to lie in the consistent contact state with the road surface, we have $s_{1}=s_{2}=1$ at every point of time. In this case, Eq. (9) is still used with no change and the differential equations of motion of the vehicle can be deduced from Eq. (8) by setting $s_{1}=s_{2}=1$ :

$$
\left\{\begin{array}{l}
m_{b} \ddot{u}_{b}+\left(c_{T 1}+c_{T 2}\right) \dot{u}_{b}+\left(c_{T 1} a_{1}-c_{T 2} a_{2}\right) \dot{\varphi}_{b}-c_{T 1} \dot{u}_{C 1}-c_{T 2} \dot{u}_{C 2} \\
\quad+\left(k_{T 1}+k_{T 2}\right) u_{b}+\left(k_{T 1} a_{1}-k_{T 2} a_{2}\right) \varphi_{b}-k_{T 1} u_{C 1}-k_{T 2} u_{C 2}=-m_{b} g, \\
J_{b} \ddot{\varphi}_{b}+\left(c_{T 1} a_{1}-c_{T 2} a_{2}\right) \dot{u}_{b}+\left(c_{T 1} a_{1}^{2}+c_{T 2} a_{2}^{2}\right) \dot{\varphi}_{b}-c_{T 1} a_{1} \dot{u}_{C 1}+c_{T 2} a_{2} \dot{u}_{C 2} \\
\quad+\left(k_{T 1} a_{1}-k_{T 2} a_{2}\right) u_{b}+\left(k_{T 1} a_{1}^{2}+k_{T 2} a_{2}^{2}\right) \varphi_{b}-k_{T 1} a_{1} u_{C 1}+k_{T 2} a_{2} u_{C 2}=0, \\
\quad m_{C 1} \ddot{u}_{C 1}-c_{T 1} \dot{u}_{b}-c_{T 1} a_{1} \dot{\varphi}_{b}+\left(c_{T 1}+c_{L 1}\right) \dot{u}_{C 1}-k_{T 1} u_{b}-k_{T 1} a_{1} \varphi_{b}+\left(k_{T 1}+k_{L 1}\right) u_{C 1} \\
\quad=-m_{C 1} g+\left[k_{L 1}\left(w_{D 1}+r_{D 1}\right)+c_{L 1}\left(\dot{w}_{D 1}+\dot{r}_{D 1}\right)\right], \\
m_{C 2} \ddot{u}_{C 2}-c_{T 2} \dot{u}_{b}+c_{T 2} a_{2} \dot{\varphi}_{b}+\left(c_{T 2}+c_{L 2}\right) \dot{u}_{C 2}-k_{T 2} u_{b}+k_{T 2} a_{2} \varphi_{b}+\left(k_{T 2}+k_{L 2}\right) u_{C 2} \\
\quad=-m_{C 2} g+\left[k_{L 2}\left(w_{D 2}+r_{D 2}\right)+c_{L 2}\left(\dot{w}_{D 2}+\dot{r}_{D 2}\right)\right] .
\end{array}\right.
$$

\subsubsection{The case of ignoring both the road deformation and the loss of contact}

If both the road deformation and the loss of contact are ignored, the differential equations of motion of the vehicle-road coupled system reduce to those of the vehicle in which $s_{1}=s_{2}=1$ and $w_{D 1}=w_{D 2}=\dot{w}_{D 1}=\dot{w}_{D 2}=0$ :

$$
\left\{\begin{array}{l}
m_{b} \ddot{u}_{b}+\left(c_{T 1}+c_{T 2}\right) \dot{u}_{b}+\left(c_{T 1} a_{1}-c_{T 2} a_{2}\right) \dot{\varphi}_{b}-c_{T 1} \dot{u}_{C 1}-c_{T 2} \dot{u}_{C 2} \\
\quad+\left(k_{T 1}+k_{T 2}\right) u_{b}+\left(k_{T 1} a_{1}-k_{T 2} a_{2}\right) \varphi_{b}-k_{T 1} u_{C 1}-k_{T 2} u_{C 2}=-m_{b} g, \\
J_{b} \ddot{\varphi}_{b}+\left(c_{T 1} a_{1}-c_{T 2} a_{2}\right) \dot{u}_{b}+\left(c_{T 1} a_{1}^{2}+c_{T 2} a_{2}^{2}\right) \dot{\varphi}_{b}-c_{T 1} a_{1} \dot{u}_{C 1}+c_{T 2} a_{2} \dot{u}_{C 2} \\
\quad+\left(k_{T 1} a_{1}-k_{T 2} a_{2}\right) u_{b}+\left(k_{T 1} a_{1}^{2}+k_{T 2} a_{2}^{2}\right) \varphi_{b}-k_{T 1} a_{1} u_{C 1}+k_{T 2} a_{2} u_{C 2}=0, \\
m_{C 1} \ddot{u}_{C 1}-c_{T 1} \dot{u}_{b}-c_{T 1} a_{1} \dot{\varphi}_{b}+\left(c_{T 1}+c_{L 1}\right) \dot{u}_{C 1}-k_{T 1} u_{b}-k_{T 1} a_{1} \varphi_{b}+\left(k_{T 1}+k_{L 1}\right) u_{C 1} \\
\quad=-m_{C 1} g+\left(k_{L 1} r_{D 1}+c_{L 1} \dot{r}_{D 1}\right), \\
\quad m_{C 2} \ddot{u}_{C 2}-c_{T 2} \dot{u}_{b}+c_{T 2} a_{2} \dot{\varphi}_{b}+\left(c_{T 2}+c_{L 2}\right) \dot{u}_{C 2}-k_{T 2} u_{b}+k_{T 2} a_{2} \varphi_{b}+\left(k_{T 2}+k_{L 2}\right) u_{C 2} \\
\quad=-m_{C 2} g+\left(k_{L 2} r_{D 2}+c_{L 2} \dot{r}_{D 2}\right) .
\end{array}\right.
$$




\subsection{Transforming the differential equations of motion into a system of all ODEs}

Because of the presence of partial differential Eq. (9), the differential equations of motion of the mechanical system cannot be able to solve. In order to obtain the functions which reflect vibrations of the automobile and the road, the Bubnov-Galerkin's method is applied here to transform the original differential equations of motion into a system of all ordinary differential equations (ODEs) of time variable only. These ODEs are called as the transformed differential equations of motion which can be solved numerically, for instance.

A procedure for reaching the purpose is given as follows:

1) Approximating the displacement expression of the beam as a series of functions which satisfy the boundary conditions Eq. (10) as:

$w(x, t)=\sum_{l=1}^{N} T_{l}(t) \sin \frac{(2 l-1) \pi x}{L}$,

where $T_{l}(t)$ are time functions to be found and $N$ is the number of terms in the series used.

Note that functions $\sin \frac{(2 l-1) \pi x}{L}$ are linearly independent and have the orthogonality:

$\int_{0}^{L} \sin \frac{(2 l-1) \pi x}{L} \sin \frac{\left(2 l^{\prime}-1\right) \pi x}{L} d x=\left\{\begin{array}{l}0, \quad l \neq l^{\prime}, \\ L / 2, \quad l=l^{\prime} .\end{array}\right.$

2) Substituting the expression of $w(x, t)$ according to Eq. (14) into Eq. (9) to obtain its derivative equation which can be written as:

$$
\begin{aligned}
\rho h_{B} & \sum_{l=1}^{N} \ddot{T}_{l}(t) \sin \frac{(2 l-1) \pi x}{L}+c_{S} \sum_{l=1}^{N} \dot{T}_{l}(t) \sin \frac{(2 l-1) \pi x}{L}+k_{S} \sum_{l=1}^{N} T_{l}(t) \sin \frac{(2 l-1) \pi x}{L} \\
& +\frac{E I}{b_{B}} \sum_{l=1}^{N} T_{l}(t) \frac{(2 l-1)^{4} \pi^{4}}{L^{4}} \sin \frac{(2 l-1) \pi x}{L}+p(x, t)=-\rho g h_{B} .
\end{aligned}
$$

3) Accepting the expression of $p(x, t)$ as $p(x, t)=\bar{p}(t) U(x)$ (variable separation method). Concretely, $p(x, t)=\bar{p}_{1}(t) U_{1}(x)$ in the contact area of front wheel and $p(x, t)=\bar{p}_{2}(t) U_{2}(x)$ in the contact area of rear wheel where $\bar{p}_{j}(t)(j=1,2)$ are time functions to be found, and $U_{j}(x)$ are the $x$-variable functions whose expressions are chosen according to the assumption of pressure distribution on the contact areas. Four types of pressure distribution proposed by the authors of this paper are presented in [16]:

- Even (constant, or rectangle) distribution: $U_{j}(x)=1(\forall x)$.

- Parabolic distribution: $U_{j}(x)=1-\left(2 x / d_{c j}\right)^{2}$.

- Cosine distribution: $U_{j}(x)=\cos \left(\pi x / d_{c j}\right)$.

- Squared cosine distribution: $U_{j}(x)=\cos ^{2}\left(\pi x / d_{c j}\right)=0.5\left[1+\cos \left(2 \pi x / d_{c j}\right)\right]$.

4) Consecutively taking $k=1,2, \ldots, N$ and multiplying both sides of Eq. (16) by $\sin \frac{(2 k-1) \pi x}{L}$, then integrating two sides of the obtained equation with respect to $x$-variable from 0 to $L$ (over the length of the beam), noting the orthogonality Eq. (15), one can get a system of $N$ ordinary differential equations of the form: 


$$
\begin{aligned}
& \rho h_{B} \ddot{T}_{k}(t)+c_{S} \dot{T}_{k}(t)+\left[k_{S}+\frac{E I}{b_{B}} \frac{(2 k-1)^{4} \pi^{4}}{L^{4}}\right] T_{k}(t)+\frac{2 I_{1 k}}{L} \bar{p}_{1}(t)+\frac{2 I_{2 k}}{L} \bar{p}_{2}(t) \\
& \quad=-\frac{4 \rho g h_{B}}{(2 k-1) \pi}
\end{aligned}
$$

where:

$$
\begin{aligned}
& I_{1 k}=\int_{I_{D 1}-0,5 d_{c 1}}^{x_{D 1}+0.5 d_{c 1}} U_{1}(x) \sin \frac{(2 k-1) \pi x}{L} d x, \\
& I_{2 k}=\int_{x_{D 2}-0.5 d_{c 2}}^{x_{c 2}} U_{2}(x) \sin \frac{(2 k-1) \pi x}{L} d x .
\end{aligned}
$$

5) Expressing functions $\bar{p}_{j}(t)(j=1,2)$ in Eq. (17) in terms of the unknown functions of time which consist of the vehicle generalized coordinates $u_{b}, \varphi_{b}, u_{C 1}, u_{C 2}$ and functions $T_{l}(t)$, $l=1,2, \ldots, N$. To reach the purpose, we use the equilibrium equations presented before:

$F_{L j}=R_{j}, \quad(j=1,2)$,

where $F_{L j}$ is the resultant force of the $j$-th wheel spring-damper couple (the contact force of the $j$-th wheel) and $R_{j}$ is the road reaction force at this wheel.

Using the expression of $w(x, t)$ in Eq. (14), we determine the vertical displacements of expected contact points $D_{j}(j=1,2)$ as:

$$
\left\{\begin{array}{l}
w_{D j}=w(x, t) \|_{x=x_{D j}}=\sum_{l=1}^{N} T_{l}(t) \sin \frac{(2 l-1) \pi x_{D j}}{L}=\sum_{l=1}^{N} \chi_{l}^{(j)} T_{l}(t), \\
\dot{w}_{D j}=\frac{\partial w(x, t)}{\partial t} \|_{x=x_{D j}}=\sum_{l=1}^{N} \dot{T}_{l}(t) \sin \frac{(2 l-1) \pi x_{D j}}{L}=\sum_{l=1}^{N} \chi_{l}^{(j)} \dot{T}_{l}(t),
\end{array}\right.
$$

where:

$\chi_{l}^{(j)}=\sin \frac{(2 l-1) \pi x_{D j}}{L}, \quad l=1, \ldots, N, \quad j=1,2$.

The expressions of $F_{L j}$ can be then obtained by putting Eq. (20) into the two last equations in Eq. (7) as:

$F_{L j}=s_{j}\left[k_{L j}\left(\sum_{l=1}^{N} \chi_{l}^{(j)} T_{l}(t)+r_{D j}-u_{C j}\right)+c_{L j}\left(\sum_{l=1}^{N} \chi_{l}^{(j)} \dot{T}_{l}(t)+\dot{r}_{D j}-\dot{u}_{C j}\right)\right]$,

$(j=1,2)$.

The reaction force $R_{j}$ can be determined by using the pressure distribution function:

$R_{j}=\int_{d_{c j}} p_{j}(x, t) b_{L} d x=\int_{-0.5 d_{c j}}^{0.5 d_{c j}} \bar{p}_{j}(t) U_{j}(x) b_{L} d x=\bar{p}_{j}(t) I_{U j} b_{L}, \quad(j=1,2)$, 
where:

$I_{U j}=\int_{-0.5 d_{c j}}^{0.5 d_{c j}} U_{j}(x) d x, \quad(j=1,2)$.

Using Eq. (22), (23) and (19), we can deduce the expressions of $\bar{p}_{j}(t)$ :

$\bar{p}_{j}(t)=\frac{s_{j}}{I_{U j} b_{L}}\left[k_{L j}\left(\sum_{l=1}^{N} \chi_{l}^{(j)} T_{l}(t)+r_{D j}-u_{C j}\right)+c_{L j}\left(\sum_{l=1}^{N} \chi_{l}^{(j)} \dot{T}_{l}(t)+\dot{r}_{D j}-\dot{u}_{C j}\right)\right]$,

$(j=1,2)$.

6) Substituting the expressions of $\bar{p}_{j}(t)$ from Eq. (25) into Eq. (17) to obtain $N$ ordinary differential equations as:

$$
\begin{aligned}
& \rho h_{B} \ddot{T}_{k}(t)+c_{S} \dot{T}_{k}(t)+\left[k_{S}+\frac{E I(2 k-1)^{4} \pi^{4}}{b_{B} L^{4}}\right] T_{k}(t) \\
& \quad+\frac{2 s_{1} I_{1 k}}{I_{U 1} b_{L} L}\left[k_{L 1}\left(\sum_{l=1}^{N} \chi_{l}^{(1)} T_{l}(t)+r_{D 1}-u_{C 1}\right)+c_{L 1}\left(\sum_{l=1}^{N} \chi_{l}^{(1)} \dot{T}_{l}(t)+\dot{r}_{D 1}-\dot{u}_{C 1}\right)\right] \\
& \quad+\frac{2 s_{2} I_{2 k}}{I_{U 2} b_{L} L}\left[k_{L 2}\left(\sum_{l=1}^{N} \chi_{l}^{(2)} T_{l}(t)+r_{D 2}-u_{C 2}\right)+c_{L 2}\left(\sum_{l=1}^{N} \chi_{l}^{(2)} \dot{T}_{l}(t)+\dot{r}_{D 2}-\dot{u}_{C 2}\right)\right] \\
& \quad=-\frac{4 \rho g h_{B}}{(2 k-1) \pi}, \quad(k=1,2, \ldots, N) .
\end{aligned}
$$

By introducing the following notations:

$\mu_{1 k}=\frac{2 s_{1} I_{1 k}}{I_{U 1} b_{L} L}, \quad \mu_{2 k}=\frac{2 s_{2} I_{2 k}}{I_{U 2} b_{L} L}, \quad H_{k}=k_{S}+\frac{E I(2 k-1)^{4} \pi^{4}}{b_{B} L^{4}}$,

$\delta_{k l}= \begin{cases}1, & k=l, \\ 0, & k \neq l,\end{cases}$

where $\delta_{k l}$ is the Cronecker's operator, Eq. (26) can be rewritten as:

$$
\begin{aligned}
\rho h_{B} \ddot{T}_{k}(t)-\mu_{1 k} c_{L 1} \dot{u}_{C 1}-\mu_{2 k} c_{L 2} \dot{u}_{C 2}+\sum_{l=1}^{N}\left(\delta_{k l} c_{S}+\mu_{1 k} c_{L 1} \chi_{l}^{(1)}+\mu_{2 k} c_{L 2} \chi_{l}^{(2)}\right) \dot{T}_{l}(t) \\
-\mu_{1 k} k_{L 1} u_{C 1}-\mu_{2 k} k_{L 2} u_{C 2}+\sum_{l=1}^{N}\left(\delta_{k l} H_{k}+\mu_{1 k} k_{L 1} \chi_{l}^{(1)}+\mu_{2 k} k_{L 2} \chi_{l}^{(2)}\right) T_{l}(t) \\
=-\frac{4 \rho g h_{B}}{(2 k-1) \pi}-\mu_{1 k}\left(k_{L 1} r_{D 1}+c_{L 1} \dot{r}_{D 1}\right)-\mu_{2 k}\left(k_{L 2} r_{D 2}+c_{L 2} \dot{r}_{D 2}\right), \quad(k=1,2, \ldots, N) .
\end{aligned}
$$

7) Using the Eq. (20) of $w_{D j}$ and $\dot{w}_{D j}$, we can rewrite the differential equations of motion of the automobile Eq. (8) in the following forms: 


$$
\begin{aligned}
& \left\{\begin{array}{l}
m_{b} \ddot{u}_{b}+\left(c_{T 1}+c_{T 2}\right) \dot{u}_{b}+\left(c_{T 1} a_{1}-c_{T 2} a_{2}\right) \dot{\varphi}_{b}-c_{T 1} \dot{u}_{C 1}-c_{T 2} \dot{u}_{C 2} \\
\quad+\left(k_{T 1}+k_{T 2}\right) u_{b}+\left(k_{T 1} a_{1}-k_{T 2} a_{2}\right) \varphi_{b}-k_{T 1} u_{C 1}-k_{T 2} u_{C 2}=-m_{b} g \\
J_{b} \ddot{\varphi}_{b}+\left(c_{T 1} a_{1}-c_{T 2} a_{2}\right) \dot{u}_{b}+\left(c_{T 1} a_{1}^{2}+c_{T 2} a_{2}^{2}\right) \dot{\varphi}_{b}-c_{T 1} a_{1} \dot{u}_{C 1}+c_{T 2} a_{2} \dot{u}_{C 2} \\
\quad+\left(k_{T 1} a_{1}-k_{T 2} a_{2}\right) u_{b}+\left(k_{T 1} a_{1}^{2}+k_{T 2} a_{2}^{2}\right) \varphi_{b}-k_{T 1} a_{1} u_{C 1}+k_{T 2} a_{2} u_{C 2}=0 \\
m_{C 1} \ddot{u}_{C 1}-c_{T 1} \dot{u}_{b}-c_{T 1} a_{1} \dot{\varphi}_{b}+\left(c_{T 1}+s_{1} c_{L 1}\right) \dot{u}_{C 1}-s_{1} c_{L 1} \sum_{l=1}^{N} \chi_{l}^{(1)} \dot{T}_{l}(t)-k_{T 1} u_{b} \\
\quad-k_{T 1} a_{1} \varphi_{b}+\left(k_{T 1}+s_{1} k_{L 1}\right) u_{C 1}-s_{1} k_{L 1} \sum_{l=1}^{N} \chi_{l}^{(1)} T_{l}(t)=-m_{C 1} g+s_{1}\left(k_{L 1} r_{D 1}+c_{L 1} \dot{r}_{D 1}\right),
\end{array}\right. \\
& m_{C 2} \ddot{u}_{C 2}-c_{T 2} \dot{u}_{b}+c_{T 2} a_{2} \dot{\varphi}_{b}+\left(c_{T 2}+s_{2} c_{L 2}\right) \dot{u}_{C 2}-s_{2} c_{L 2} \sum_{l=1}^{N} \chi_{l}^{(2)} \dot{T}_{l}(t)-k_{T 2} u_{b} \\
& +k_{T 2} a_{2} \varphi_{b}+\left(k_{T 2}+s_{2} k_{L 2}\right) u_{C 2}-s_{2} k_{L 2} \sum_{l=1}^{N} \chi_{l}^{(2)} T_{l}(t)=-m_{C 2} g+s_{2}\left(k_{L 2} r_{D 2}+c_{L 2} \dot{r}_{D 2}\right) \text {. }
\end{aligned}
$$

Now the original differential equations of motion of the vehicle-road coupled system with the presence of partial differential Eq. (9) have been transformed into a system of $(4+N)$ ordinary differential Eqs. (29) and (28).

The transformed differential equations of motion Eqs. (29) and (28) can be written in matrix form as:

$[M] \ddot{\vec{q}}+[C] \dot{\vec{q}}+[K] \vec{q}=\vec{F}$,

where $\vec{q}$ - vector of generalized coordinates, $\vec{F}$ - vector of excitation; $[M],[C],[K]-$ mass, damping and stiffness matrices, respectively.

Concrete forms of the vectors and matrices mentioned above are determined as follows:

- The vector of generalized coordinates has $(4+N)$ elements as:

$\vec{q}=\left[u_{b}(t), \varphi_{b}(t), u_{C 1}(t), u_{C 2}(t), T_{1}(t), T_{2}(t), T_{3}(t), \ldots, T_{N}(t)\right]^{T}$.

- The vector of excitation is expressed as:

$$
\vec{F}=\left[\begin{array}{c}
-m_{b} g \\
0 \\
-m_{C 1} g+s_{1}\left(k_{L 1} r_{D 1}+c_{L 1} \dot{r}_{D 1}\right) \\
-m_{C 2} g+s_{2}\left(k_{L 2} r_{D 2}+c_{L 2} \dot{r}_{D 2}\right) \\
-\frac{4 \rho g h_{B}}{1 \pi}-\mu_{11}\left(k_{L 1} r_{D 1}+c_{L 1} \dot{r}_{D 1}\right)-\mu_{21}\left(k_{L 2} r_{D 2}+c_{L 2} \dot{r}_{D 2}\right) \\
-\frac{4 \rho g h_{B}}{3 \pi}-\mu_{12}\left(k_{L 1} r_{D 1}+c_{L 1} \dot{r}_{D 1}\right)-\mu_{22}\left(k_{L 2} r_{D 2}+c_{L 2} \dot{r}_{D 2}\right) \\
-\frac{4 \rho g h_{B}}{5 \pi}-\mu_{13}\left(k_{L 1} r_{D 1}+c_{L 1} \dot{r}_{D 1}\right)-\mu_{23}\left(k_{L 2} r_{D 2}+c_{L 2} \dot{r}_{D 2}\right) \\
\cdots \\
-\frac{4 \rho g h_{B}}{(2 N-1) \pi}-\mu_{1 N}\left(k_{L 1} r_{D 1}+c_{L 1} \dot{r}_{D 1}\right)-\mu_{2 N}\left(k_{L 2} r_{D 2}+c_{L 2} \dot{r}_{D 2}\right)
\end{array}\right]
$$

- The mass matrix is a diagonal matrix as: 


$$
[M]=\left[\begin{array}{ccccccccc}
m_{b} & 0 & 0 & 0 & 0 & 0 & 0 & \cdots & 0 \\
0 & J_{b} & 0 & 0 & 0 & 0 & 0 & \cdots & 0 \\
0 & 0 & m_{C 1} & 0 & 0 & 0 & 0 & \cdots & 0 \\
0 & 0 & 0 & m_{C 2} & 0 & 0 & 0 & \cdots & 0 \\
0 & 0 & 0 & 0 & \rho h_{B} & 0 & 0 & \cdots & 0 \\
0 & 0 & 0 & 0 & 0 & \rho h_{B} & 0 & \cdots & 0 \\
0 & 0 & 0 & 0 & 0 & 0 & \rho h_{B} & \cdots & 0 \\
\cdots & \ldots & \ldots & \ldots & \ldots & \ldots & \ldots & \cdots & \ldots \\
0 & 0 & 0 & 0 & 0 & 0 & 0 & \cdots & \rho h_{B}
\end{array}\right] .
$$

- The stiffness matrix $[K]$ has $(4+N)$ rows, each of which has $(4+N)$ elements as:

$$
\begin{aligned}
& {\left[K_{1 i}\right]=\left[k_{T 1}+k_{T 2},-k_{T 1} a_{1}+k_{T 2} a_{2},-k_{T 1},-k_{T 2}, 0,0,0, \ldots, 0\right],} \\
& {\left[K_{2 i}\right]=\left[-k_{T 1} a_{1}+k_{T 2} a_{2}, k_{T 1} a_{1}^{2}+k_{T 2} a_{2}^{2},-k_{T 1} a_{1}, k_{T 2} a_{2}, 0,0,0, \ldots, 0\right],} \\
& {\left[K_{3 i}\right]} \\
& \quad=\left[-k_{T 1},-k_{T 1} a_{1}, k_{T 1}+s_{1} k_{L 1}, 0,-s_{1} \chi_{1}^{(1)} k_{L 1},-s_{1} \chi_{2}^{(1)} k_{L 1},-s_{1} \chi_{3}^{(1)} k_{L 1}, \ldots,-s_{1} \chi_{N}^{(1)} k_{L 1}\right], \\
& {\left[K_{4 i}\right]=\left[-k_{T 2}, k_{T 2} a_{2}, 0, k_{T 2}+s_{2} k_{L 2},-s_{2} \chi_{1}^{(2)} k_{L 2},-s_{2} \chi_{2}^{(2)} k_{L 2},-s_{2} \chi_{3}^{(2)} k_{L 2}, \ldots,-s_{2} \chi_{N}^{(2)} k_{L 2}\right],} \\
& {\left[K_{5 i}\right]=\left[0,0,-\mu_{11} k_{L 1},-\mu_{12} k_{L 2}, H_{1}+\mu_{11} k_{L 1} \chi_{1}^{(1)}+\mu_{12} k_{L 2} \chi_{1}^{(2)}, \mu_{11} k_{L 1} \chi_{2}^{(1)}+\mu_{12} k_{L 2} \chi_{2}^{(2)},\right.} \\
& \left.\quad \mu_{11} k_{L 1} \chi_{3}^{(1)}+\mu_{12} k_{L 2} \chi_{3}^{(2)}, \ldots, \mu_{11} k_{L 1} \chi_{N}^{(1)}+\mu_{12} k_{L 2} \chi_{N}^{(2)}\right], \\
& {\left[K_{6 i}\right]=\left[0,0,-\mu_{21} k_{L 1},-\mu_{22} k_{L 2}, \mu_{21} k_{L 1} \chi_{1}^{(1)}+\mu_{22} k_{L 2} \chi_{1}^{(2)}, H_{2}+\mu_{21} k_{L 1} \chi_{2}^{(1)}+\mu_{22} k_{L 2} \chi_{2}^{(2)},\right.} \\
& \left.\mu_{21} k_{L 1} \chi_{3}^{(1)}+\mu_{22} k_{L 2} \chi_{3}^{(2)}, \ldots, \mu_{21} k_{L 1} \chi_{N}^{(1)}+\mu_{22} k_{L 2} \chi_{N}^{(2)}\right], \\
& {\left[K_{4+N, i}\right]=\left[0,0,-\mu_{N 1} k_{L 1},-\mu_{N 2} k_{L 2}, \mu_{N 1} k_{L 1} \chi_{1}^{(1)}+\mu_{N 2} k_{L 2} \chi_{1}^{(2)}, \mu_{N 1} k_{L 1} \chi_{2}^{(1)}+\mu_{N 2} k_{L 2} \chi_{2}^{(2)}\right.} \\
& \left.\mu_{N 1} k_{L 1} \chi_{3}^{(1)}+\mu_{N 2} k_{L 2} \chi_{3}^{(2)}, \ldots, H_{N}+\mu_{N 1} k_{L 1} \chi_{N}^{(1)}+\mu_{N 2} k_{L 2} \chi_{N}^{(2)}\right] .
\end{aligned}
$$

- The damping matrix $[C]$ has the form similar to that of the stiffness matrix. One can obtain matrix $[C]$ from matrix $[K]$ by replacing the notations $\left\{H_{k}, k_{T 1}, k_{T 2}, k_{L 1}, k_{L 2}\right\}$ by the notations $\left\{c_{S}, c_{T 1}, c_{T 2}, c_{L 1}, c_{L 2}\right\}$, respectively.

\subsection{Initial conditions}

The common form of initial conditions in problems on the vibration of automobile is that the vehicle is moving on a horizontal road with the surface completely smooth when entering a rough surface, and the initial time point $(t=0)$ is chosen so that the vehicle is still not entering or starts entering the rough road surface. At that point of time, vibration of the vehicle does not appear, thus the vectors of generalized velocities and accelerations are equal to zeros and the vector of generalized coordinates is equal to that of static displacements of the system:

$\ddot{\vec{q}}\left\|_{t=0}=\ddot{\overrightarrow{q_{0}}}=\overrightarrow{0}, \quad \dot{\vec{q}}\right\|_{t=0}=\dot{\overrightarrow{q_{0}}}=\overrightarrow{0}, \quad \vec{q} \|_{t=0}=\vec{q}_{0}$.

The vector of static displacements $\vec{q}_{0}$ can be determined by using data Eq. (35) into the transformed differential equations of motion Eq. (30) and deducing:

$[K]_{0} \vec{q}_{0}=\vec{F}_{0} \Rightarrow \vec{q}_{0}=[K]_{0}^{-1} \vec{F}_{0}$,

where $[K]_{0}, \vec{F}_{0}-$ initial values of the stiffness matrix $[K]$ and the excitation vector $\vec{F}$.

In order to calculate the values of the elements of matrix $[K]_{0}$, we firstly make and solve the static equilibrium equations of the automobile to get the initial values of contact forces $F_{L j}^{(0)}$ and reaction forces from $\operatorname{road} R_{j}^{(0)}(j=1,2)$ as: 
$F_{L 1}^{(0)}=R_{1}^{(0)}=\left(m_{C 1}+m_{b} \frac{a_{2}}{a_{1}+a_{2}}\right) g, \quad F_{L 2}^{(0)}=R_{2}^{(0)}=\left(m_{C 2}+m_{b} \frac{a_{1}}{a_{1}+a_{2}}\right) g$.

The obtained values of two contact forces allow to calculate the initial values of static deformations of the springs which represent the two wheels $\Delta z_{L j}^{(0)}$, the lengths in $x$-direction of two contact areas $d_{c j}^{(0)}$ as follows:

$\Delta z_{L 1}^{(0)}=F_{L 1}^{(0)} / k_{L 1}, \quad d_{c 1}^{(0)}=2 \sqrt{r_{w}^{2}-\left(r_{w}-\Delta z_{L 1}^{(0)}\right)^{2}}$,

$\Delta z_{L 2}^{(0)}=F_{L 2}^{(0)} / k_{L 2}, \quad d_{c 2}^{(0)}=2 \sqrt{r_{w}^{2}-\left(r_{w}-\Delta z_{L 2}^{(0)}\right)^{2}}, \quad j=1,2$.

Now we can calculate the initial values of the integrations in Eq. (24) and (18).

Vector $\vec{F}_{0}$ can be obtained from vector $\vec{F}$ simply by assigning value zero to all quantities $r_{D 1}$, $r_{D 2}, \dot{r}_{D 1}$ and $\dot{r}_{D 2}$.

\section{Some results from numerical computation}

This section presents some illustrating results obtained from numerical computation when the initial conditions mentioned in Section 2.8 are applied. The results we can directly obtain from the process of computation consist of the generalized coordinates, velocities, accelerations as functions of time as listed below:

$u_{b}(t), \varphi_{b}(t), u_{C 1}(t), u_{C 2}(t), T_{1}(t), T_{2}(t), \ldots, T_{N}(t)$,

$\dot{u}_{b}(t), \dot{\varphi}_{b}(t), \dot{u}_{C 1}(t), \dot{u}_{C 2}(t), \dot{T}_{1}(t), \dot{T}_{2}(t), \ldots, \dot{T}_{N}(t)$,

$\ddot{u}_{b}(t), \ddot{\varphi}_{b}(t), \ddot{u}_{C 1}(t), \ddot{u}_{C 2}(t), \ddot{T}_{1}(t), \ddot{T}_{2}(t), \ldots, \ddot{T}_{N}(t)$.

Basing on these results, one can additionally obtain the contact forces $F_{L j}(t)(j=1,2)$ as time functions, the total time of contact loss in a given interval of computation time and fulfil other desired considerations.

The input data used for numerical calculation are taken as follows:

- The values of vehicle parameters are those of automobile GAZ-66 [19]: $a_{1}=1.563 \mathrm{~m}$, $a_{2}=1.737 \mathrm{~m}, r_{w}=0.45 \mathrm{~m}, b_{L}=0.25 \mathrm{~m}, m_{b}=2200 \mathrm{~kg}, J_{b}=2750 \mathrm{~kg} \cdot \mathrm{m}^{2}, m_{C 1}=660 \mathrm{~kg}$, $m_{C 2}=580 \mathrm{~kg}, k_{T 1}=246000 \mathrm{~N} / \mathrm{m}, k_{T 2}=196000 \mathrm{~N} / \mathrm{m}, k_{L 1}=k_{L 2}=800000 \mathrm{~N} / \mathrm{m}$, $c_{T 1}=c_{T 2}=1500 \mathrm{~N} . \mathrm{s} / \mathrm{m}, c_{L 1}=c_{L 2}=62000 \mathrm{~N} . \mathrm{s} / \mathrm{m}$.

- The values of parameters concerned with the elastic beam and the Kelvin's visco-elastic ground are referred to those in [11]: $L=160 \mathrm{~m}, b_{B}=1.00 \mathrm{~m}, h_{B}=0.30 \mathrm{~m}, E=6.998 \times 10^{9} \mathrm{~N} / \mathrm{m}^{2}$, $\rho=2373 \mathrm{~kg} / \mathrm{m}^{3}, k_{S}=8 \times 10^{6} \mathrm{~N} / \mathrm{m}^{2}, c_{S}=0.3 \times 10^{6} \mathrm{~N} . \mathrm{s} / \mathrm{m}^{2}$.

- The used values of $N(N=5)$ in series Eq. (14) is chosen from considering the convergence of calculation results while the values of geometrical parameters concerned with road surface are taken based on actual observations. The pressure distribution function applied is parabolic.

The case under consideration which is presented below concerns with the road excitation as a single pulse in which the road profile is a half cycle of sinusoidal wave. This type of road profile is described in Fig. 4 where $h_{E}$ and $L_{E}$ are respectively the height and length of the pulse. The values of $h_{E}$ and $L_{E}$ are taken as $h_{E}=0.12 \mathrm{~m}, L_{E}=0.65 \mathrm{~m}$. The distance $x_{0}$ in Fig. 4 is introduced for the generality of consideration and the clarity of graphs in time domain. The value of $x_{0}$ is calculated in accordance with a specified value of time $\left(t_{0}\right)$ and vehicle velocity $(V)$ by using the formula $x_{0}=V t_{0}$ (the value $t_{0}=0.5 \mathrm{~s}$ is fixed in this paper). 


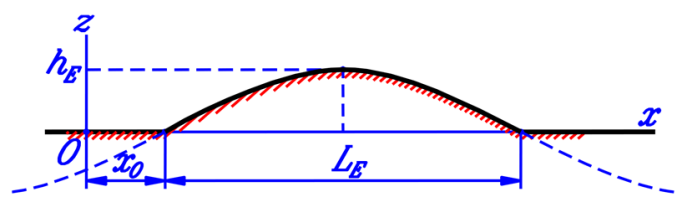

Fig. 4. Geometrical description of a half cycle of sinusoidal wave

Vibration of the vehicle will be considered in four different cases which involve the fact of taking road deformation (RD) and wheel separation (WS) into account or not. For ease of presentation, the four cases are denoted as follows:

Case 1: Taking account of both road deformation and wheel separation.

Case 2: Taking account of road deformation and ignoring wheel separation.

Case 3: Ignoring road deformation and taking account of wheel separation.

Case 4: Ignoring both road deformation and wheel separation.

\subsection{Comparison of the results obtained from four consideration cases.}

The plots in Fig. 5 and Fig. 6 present the changes in vertical displacement and acceleration of vehicle body while the plots in Fig. 7 and Fig. 8 respectively show the variation of contact forces at the front and the rear wheels with respect to time when the vehicle speed is taken as $20 \mathrm{~km} / \mathrm{h}$.

It can be seen from the plot form show that:

- Wheel separation really appear in the case of consideration. In Fig. 5, the plot of $u_{b}(t)$ has some pieces lying upper than zero level, and in Fig. 7 and Fig. 8, the plots of $F_{L 1}(t)$ and $F_{L 2}(t)$ in case 1 and case 3 have some flat pieces lying right on the abscissa.

- The plots in two cases of taking wheel separation into account (case 1 and case 3) have evident differences both in comparison with each other and with the other two cases.

- The differences in vertical displacements and accelerations between the two cases of disregarding wheel separation into account (case 2 and case 4) are not as significant as the differences in the same quantities between any other two cases. This means the effect of wheel separation on vehicle vibration is more significant than road deformation, and moreover, wheel separation makes the effect of road deformation become more significant at least in the situation of consideration.

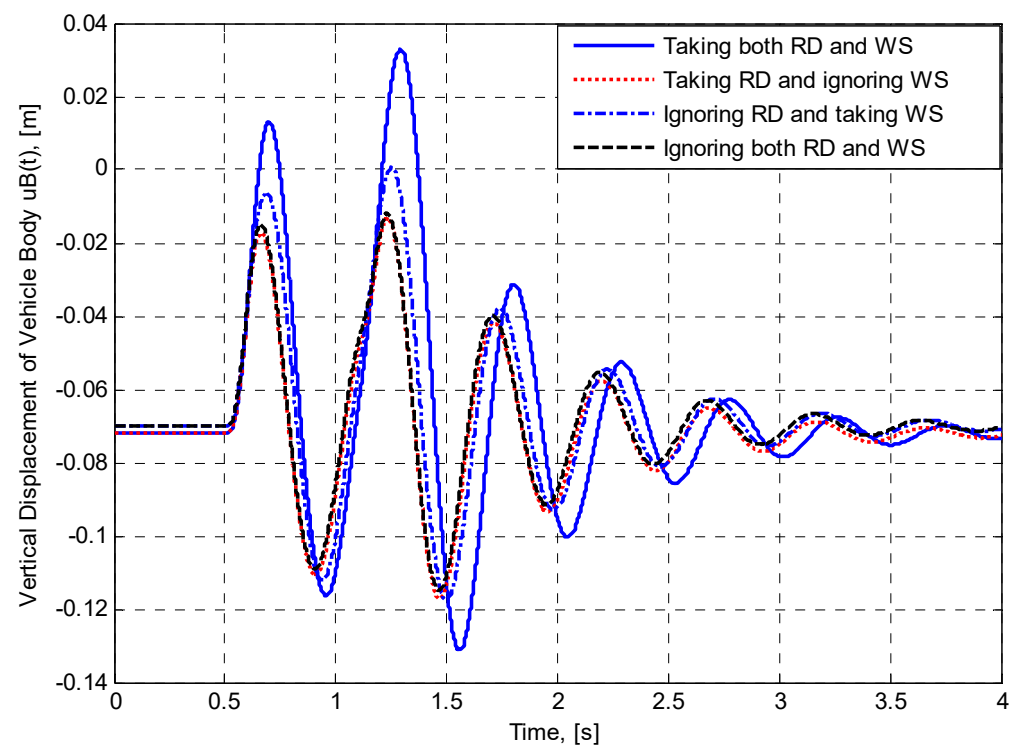

Fig. 5. The change in vertical displacement of vehicle body 


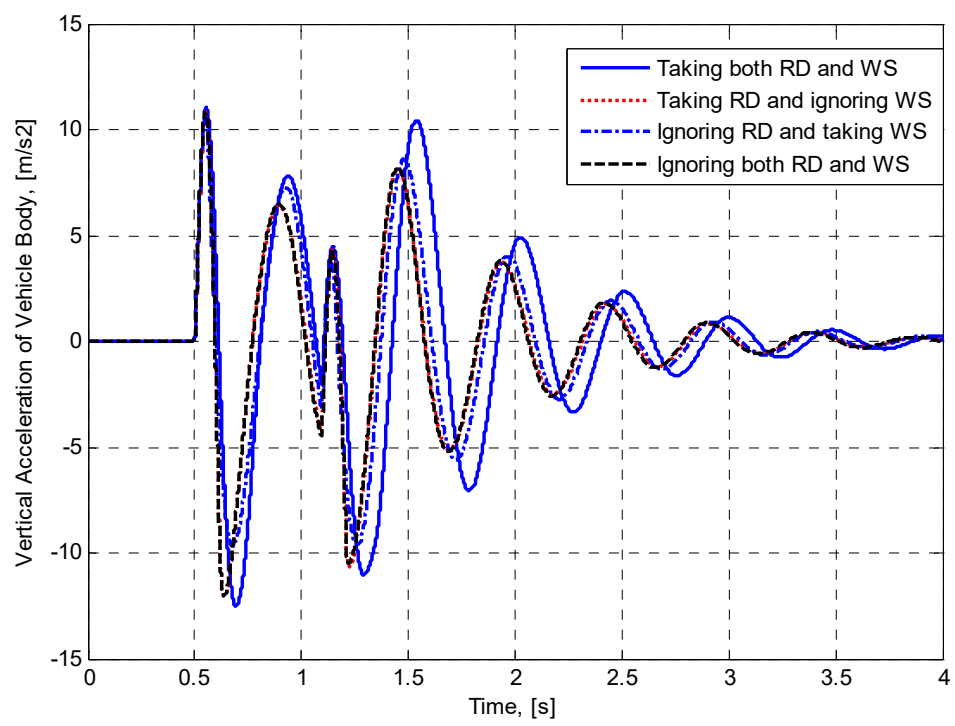

Fig. 6. The change in vertical acceleration of vehicle body

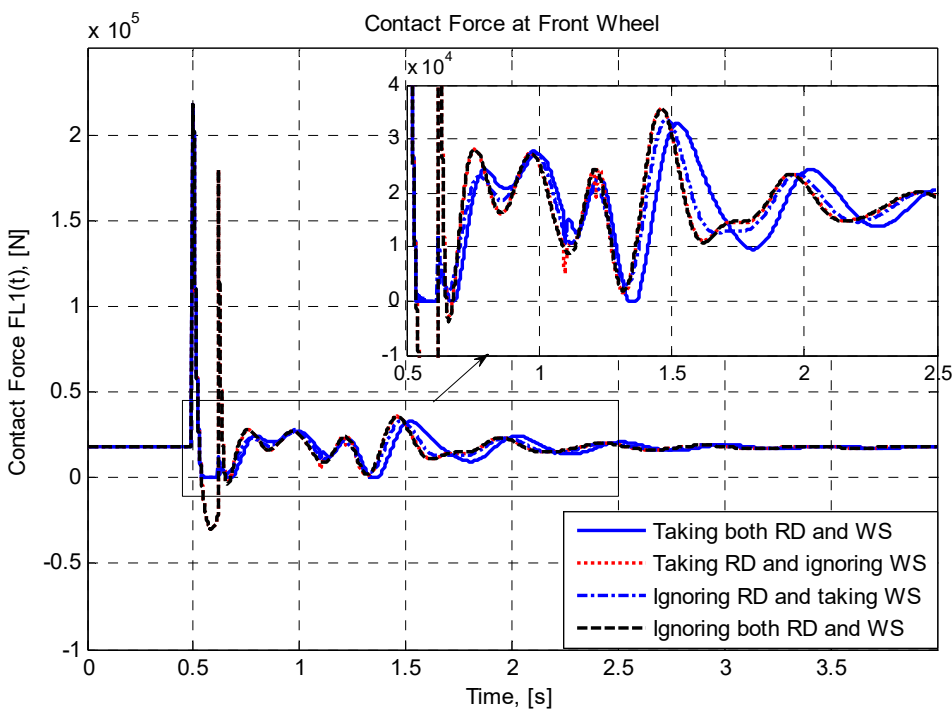

Fig. 7. Variation of contact force at the front wheel

\subsection{Effect of vehicle speed on response of the system}

Table 1 presents some numerical results which reflect the effect of vehicle speed $(V)$ on the root mean square values RMS(.) of vertical acceleration of vehicle body and contact forces at the two wheels; the maximum value of vertical displacement of vehicle body. The used values of vehicle speed are discretely taken from 0 to $35 \mathrm{~km} / \mathrm{h}$. The graphs which depict the mentioned results are describe in Fig. 9. The results also involve the four considered cases mentioned above. The first column correspond to the static state of the vehicle $(V=0)$.

The plots in Fig. 9 show that:

- There are significant differences between the two cases of taking wheel separation into account in comparison with the two cases of disregarding this phenomenon. Especially, the difference in results between the two cases of not taking the loss of contact into account is not as much as the differences between any other two cases. 
- The increase in vehicle speed leads to the increase in the RMS values of contact forces in general. The fact may involve the transformation of kinematic energy into potential energy of deformable parts.

- The effect of the increase in vehicle speed on the two other quantities (the RMS value of vertical acceleration and the maximum value of vertical displacement of vehicle body) does not follow an obvious trend. This may concerns the complexity in relations of geometrical parameters of the vehicle and the road profile and the velocity of movement.

- The RMS values of contact forces in the two cases of taking wheel separation into account are less than the corresponding values of the other two cases. This is reasonable because in periods of losing contact at any wheel, the contact force is equal to zero.

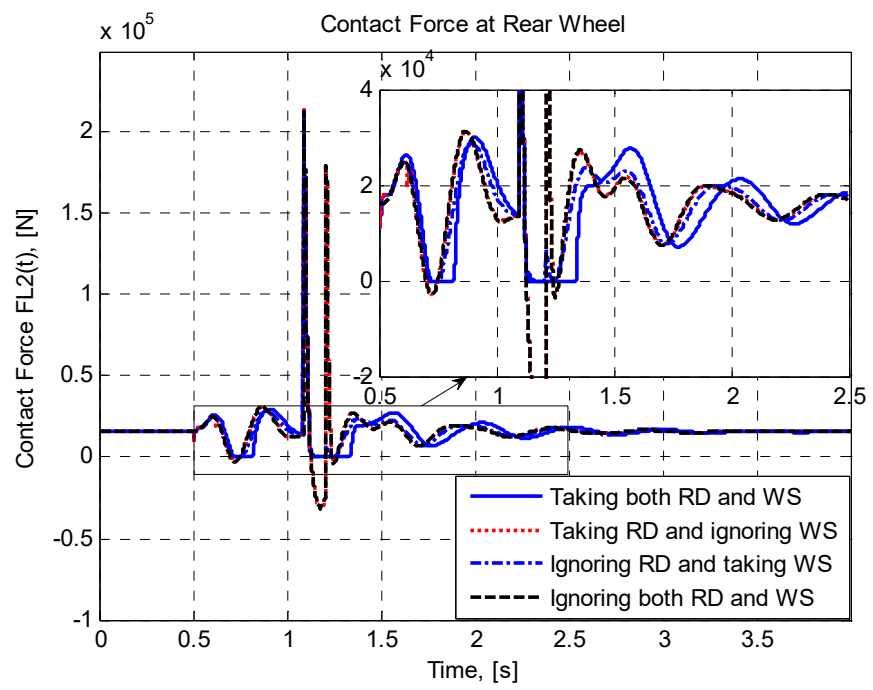

Fig. 8. Variation of contact force at the rear wheel

Table 1. Effect of vehicle speed on RMS values of some typical quantities and the total time of losing contact

\begin{tabular}{|c|c|c|c|c|c|c|c|c|c|}
\hline \multicolumn{2}{|c|}{ Vehicle speed (V), [km/h] } & 0 & 5 & 10 & 15 & 20 & 25 & 30 & 35 \\
\hline \multirow{4}{*}{$\operatorname{RMS}\left(\ddot{u}_{b}\right),\left[\mathrm{m} / \mathrm{s}^{2}\right]$} & Case 1 & 0 & 4.0779 & 4.1048 & 3.6398 & 4.2111 & 4.7999 & 4.5314 & 4.1904 \\
\hline & Case 2 & 0 & 4.0779 & 4.5836 & 3.9364 & 3.3969 & 3.4797 & 2.9569 & 2.3620 \\
\hline & Case 3 & 0 & 4.1153 & 4.1521 & 3.8054 & 3.4496 & 3.8003 & 3.3325 & 2.6553 \\
\hline & Case 4 & 0 & 4.1153 & 4.6327 & 4.0155 & 3.4103 & 3.5263 & 3.0045 & 2.3996 \\
\hline \multirow{4}{*}{$\operatorname{RMS}\left(F_{L 1}\right),[\mathrm{N}]$} & Case 1 & 17835 & 18869 & 19117 & 19514 & 20177 & 21056 & 21821 & 22654 \\
\hline & Case 2 & 17835 & 18869 & 19702 & 20301 & 21410 & 23123 & 25106 & 27101 \\
\hline & Case 3 & 17835 & 18889 & 19157 & 19560 & 20092 & 20971 & 21798 & 22542 \\
\hline & Case 4 & 17835 & 18889 & 19741 & 20373 & 21505 & 23280 & 25340 & 27427 \\
\hline \multirow{4}{*}{$\operatorname{RMS}\left(F_{L 2}\right),[\mathrm{N}]$} & Case 1 & 15912 & 16882 & 17144 & 17526 & 18362 & 19241 & 19806 & 19338 \\
\hline & Case 2 & 15912 & 16882 & 17679 & 18170 & 19532 & 21107 & 23057 & 24977 \\
\hline & Case 3 & 15912 & 16904 & 17133 & 17373 & 18223 & 19009 & 19894 & 20623 \\
\hline & Case 4 & 15912 & 16904 & 17702 & 18234 & 19621 & 21254 & 23267 & 25266 \\
\hline \multirow{4}{*}{$z_{\text {bmax }},[\mathrm{m}]$} & Case 1 & -0.0721 & 0.0284 & 0.0213 & 0.0067 & 0.0328 & 0.0445 & 0.0451 & 0.0621 \\
\hline & Case 2 & -0.0721 & 0.0284 & 0.0180 & -0.0025 & -0.0133 & -0.0093 & -0.0264 & -0.0392 \\
\hline & Case 3 & -0.0702 & 0.0302 & 0.0202 & 0.0056 & 0.0006 & 0.0066 & -0.0010 & -0.0236 \\
\hline & Case 4 & -0.0702 & 0.0302 & 0.0201 & -0.0004 & -0.0121 & -0.0069 & -0.0238 & -0.0372 \\
\hline
\end{tabular}



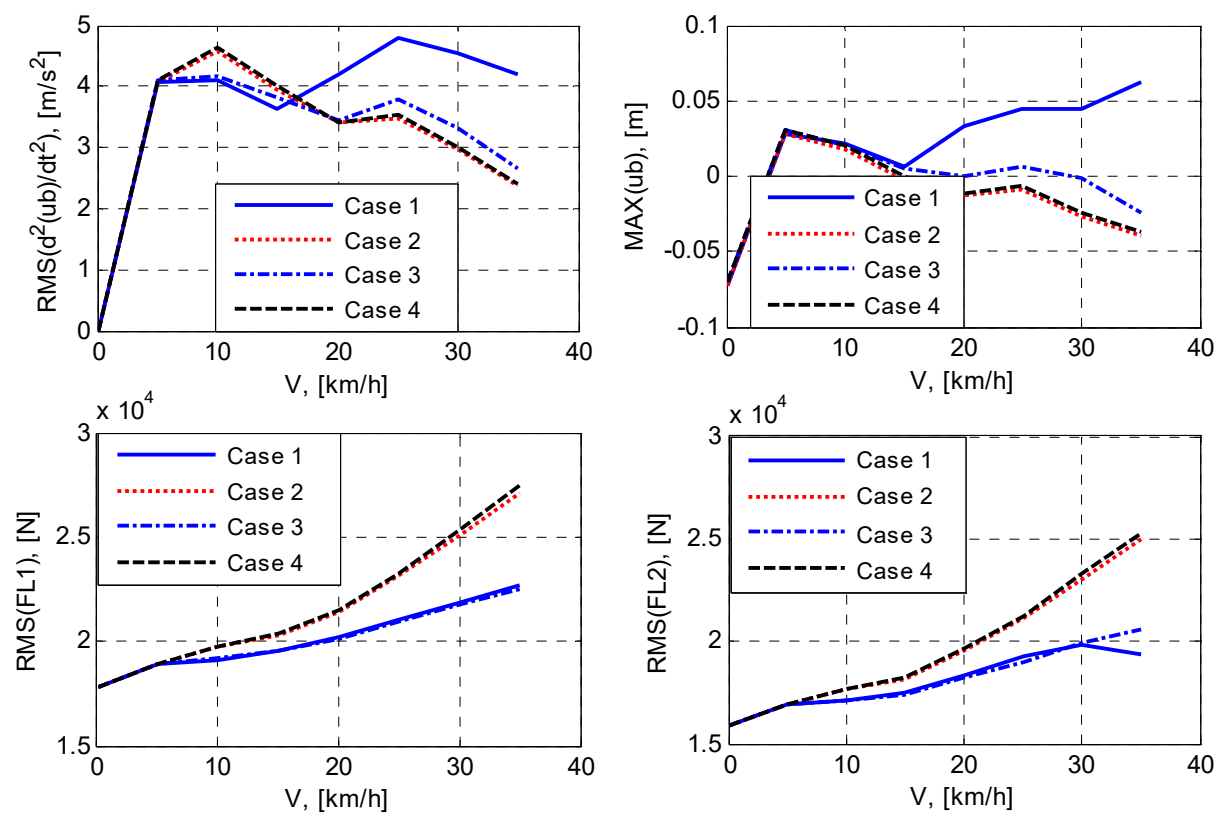

Fig. 9. Effect of vehicle speed on RMS or maximum value of some parameters reflecting vibration of the vehicle

\section{Conclusions}

The article has made a longitudinal vibration model of a two-axle automobile where both road deformation and the loss of contact are taken into account. The automobile is modeled as a linear vibration system which has three masses and four degrees of freedom. The deformed road is modeled as an elastic beam which is simply supported at the two ends and lies on the Kelvin's visco-elastic ground. The model of wheel-road contact has taken account of wheel separation and the change in dimmension of the contact area if exists. The original differential equations of motion of the vehicle-road coupled system with a partial differential equation have been transformed into a system of all ordinary differential equations by applying the Bubnov-Galerkin's method. An example of numerical computation has been fulfilled where the results of four different cases of formulation have been put in comparison and the effect of vehicle speed on vibration of the system has been considered. The obtained results proves the necessity of taking the loss of contact into account when considering vibration of vehicles.

\section{References}

[1] Bala Raju A., Venkatachalam R. Analysis of vibrations of automobile suspension system using full-car model. International Journal of Scientific and Engineering Research, Vol. 4, Issue 9, 2013, p. 2105-2111.

[2] Dung Nguyen D. Research on Dynamic Interaction in Vertical Direction between Automobiles and Roads. Ph.D. Thesis, Hanoi, Vietnam, 2018.

[3] Gao W., Zhang N., Du H. P. A half-car model for dynamic analysis of vehicles with random parameters. Proceedings of the Fifth Australasian Congress on Applied Mechanics (ACAM 2007), Brisbane Australia, Vol. 1, 2007, p. 595-600.

[4] Hu D., Yan Y., Liqun C., Shaopu Y. Vibration of vehicle - pavement coupled system based on a nonlinear foundation. Journal of Sound and Vibration, Vol. 333, Issue 24, 2014, p. 6623-6636.

[5] Jazar Reza N. Vehicle Dynamics-Theory and Application. Second Edition, Springer-Verlag New York, USA, 2014.

[6] Lap Duc V. Vibration of Automobiles. People's Army Publisher, Vietnam, 2011. 
[7] Mahmoud R., Atanu B. Passive suspension modelling and analysis of a full-car model. International Journal of Advanced Science Engineering Technology, Vol. 3, Issue 2, 2013, p. 250-261.

[8] Mitra A., Benerjee N., Khalane H. A., Sonawane M. A., Joshi D. R., Bagul G. R. Simulation and analysis of full car model for various road profile on an analytically validated MATLAB/SIMULINK model. IOSR Journal of Mechanical and Civil Engineering (IOSR-JMCE), 2013, p. 22-33.

[9] Mohd Azman A., Jazli Firdaus J., Ahmed E. M. Vehicle dynamics-modeling and simulation. first edition, centre for advanced research on energy. Technical University of Malaysia Malacca, 2016.

[10] Spinola Roberto B. Vehicle Vibration response subjected to long wave measured pavement irregularity. Journal of Mechanical Engineering and Automation, Vol. 2, Issue 2, 2012, p. 17-24.

[11] Shaopu Y., Liqun C., Shaohua L. Dynamics of Vehicle-Road Coupled Systems. First Edition, Springer-Verlag Berlin Heidelberg, 2015.

[12] Saopu Y., Saohua L., Yongjie L. Investigation on dynamical interaction between a heavy vehicle and road pavement. Vehicle System Dynamics. International Journal of Vehicle Mechanics and Mobility, Vol. 48, Issue 8, 2010, p. 923-944.

[13] Syabillah S., Samin P. M., Jamaluddin H., Rahman R. A., Burhaumudin M. S. Modelling and Validation of 7-DOF Ride Model for Heavy Vehicle. Proceedings of the International Conference on Automotive, Mechanical and Materials Engineering, 2012, p. 108-112.

[14] Ham V. C., Dung N. D. Consideration on the loss of contact between the wheels of automobile and road surface caused by vertical vibrations. Proceedings of the National Conference on Engineering Mechanics, Danang Vietnam, Vol. 2, 2015, p. 108-115.

[15] Cuong P. M., Dung T. Q., Nam L. H. Consideration on vibration of automobiles in quarter model with the loss of contact taken into account. Journal of Science and Technology, Military Technical Academy, Vietnam, Vol. 183, 2017, p. 64-73.

[16] Ham V. C., Cuong P. M., Dung T. Q. Consideration of the problem about vibration of automobiles in one fourth model with taking road deformation and the loss of contact into account. Journal of Vibroengineering, Vol. 22, Issue 4, 2020, p. 945-958.

[17] Ham V. C., Cuong P. M., Dung T. Q. Consideration on vibration of automobiles in planar model taking account of the loss of contact. Proceedings of the National Conference on Mechanics and Transportation Engineering, Vol. 2, 2017, p. 236-332.

[18] Dao Van D. Analysis on Stability and Dynamics of Structures made of Functionally Graded Materials. Publisher of Sciences and Engineerings, Vietnam, 2016.

[19] Lap V. D. Handbook for looking up Specifications of Automobiles. Military Technical Academy, Vietnam.

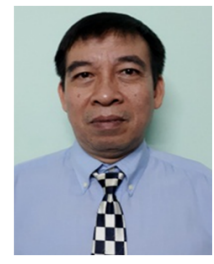

Vu Cong Ham received Ph.D. degree in applied mechanics from Le Quy Don University, Ha Noi, Viet Nam, in 2003. Now Lecturer Associate Professor in the faculty of Mechanics, Le Quy Don Technical University. His current research interests include computational mechanics, dynamics of machines and multibody systems.

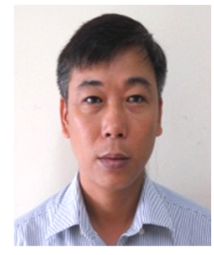

Phung Manh Cuong received master's degree in mechanical engineering from Le Quy Don University, Ha Noi, Viet Nam, in 2013. Now a Ph.D. study in the faculty of Mechanics, Le Quy Don Technical University. His current research interests include dynamics and vibration of automobile.

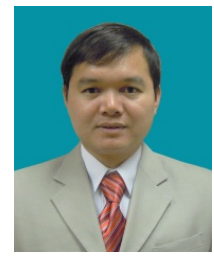

Tran Quang Dung received Ph.D. degree in mechanical engineering from Defense University, Czech Republic, in 2012. Now Lecturer Associate Professor in the faculty of Mechanics, Le Quy Don Technical University, Ha Noi, Viet Nam. My current research interests include computational mechanics, dynamics of machines and multibody systems. 